\title{
Effects of microplastic and microglass particles on soil microbial community structure in an arable soil (Chernozem)
}

\author{
Katja Wiedner ${ }^{1}$ and Steven Polifka ${ }^{2}$ \\ ${ }^{1}$ Sustainable Environmental Solutions Consulting UG (SEnSol), Gleichen, Germany \\ ${ }^{2}$ Department of Geography, \\ Ludwig-Maximilians-Universität, Munich, Germany
}

Correspondence: Katja Wiedner (kawi.science@googlemail.com)

Received: 20 June 2019 - Discussion started: 11 July 2019

Revised: 14 April 2020 - Accepted: 8 June 2020 - Published: 23 July 2020

\begin{abstract}
Microplastic and microglass particles from different sources enter aquatic and terrestrial environments. The complexity of their environmental impact is difficult to capture, and the consequences for ecosystem components, for example, the soil microorganisms, are virtually unknown. To address this issue, we performed an incubation experiment by adding $1 \%$ of five different types of impurities $(\leq 100 \mu \mathrm{m})$ to an agriculturally used soil (Chernozem) and simulating a worst-case scenario of contamination. The impurities were made of polypropylene (PP), low-density polyethylene (LDPE), polystyrene (PS), polyamide 12 (PA12) and microglass. After $80 \mathrm{~d}$ of incubation at $20^{\circ} \mathrm{C}$, we examined the soil microbial community structure by using phospholipid fatty acids (PLFAs) as markers for bacteria, fungi and protozoa. The results showed that soil microorganisms were not significantly affected by the presence of microplastic and microglass. However, PLFAs tend to increase with LDPE (28\%), PP (19\%) and microglass (11\%) in treated soil in comparison with untreated soil, whereas PLFAs in PA12 $(32 \%)$ and PS (11\%) in treated soil decreased. Interestingly, PLFAs revealed significant differences in PA12 (-89\%) and PS (-43\%) in comparison with LDPE. Furthermore, variability of bacterial PLFAs was much higher after microplastic incubation, while fungi seemed to be unaffected from different impurities after $80 \mathrm{~d}$ of incubation. Similar results were shown for protozoa, which were also more or less unaffected by microplastic treatment as indicated by the minor reduction in PLFA contents compared to the control group. In contrast, microglass seems to have an inhibiting effect on protozoa because PLFAs were under the limit of determination. Our study indicated that high amounts of different microplastics may have contrary effects on soil microbiology. Microglass might have a toxic effect for protozoa.
\end{abstract}

\section{Introduction}

Microplastics are used, for example, for a range of consumer products or in industrial application such as abrasives, filler, film and binding agents. The identification and quantification of the sources and pathways of microplastics into the environment are highly diverse and difficult to detect. While different methods have been developed for synthetic polymer identification and quantification in sediments and water, analytical methods for soil matrices are still lacking or in an early experimental stage (e.g., Hurley et al., 2018). It is assumed that microplastics enter (agricultural) soils with soil amendments, irrigation and the use of agricultural plastic films for mulch applications but also through flooding, atmospheric deposition and littering (Bläsing and Amelung, 2018; Hurley and Nizzetto, 2018; Kyrikou and Briassoulis, 2007; Ng et al., 2018; Weithmann et al., 2018). The extent of microplastics-polluted soil ecosystems is probably much higher than previously thought. For instance, a recent study by Weithmann et al. (2018) found 895 plastic particles $(>1 \mathrm{~mm})$ per kilogram of dry weight in digestate from a biowaste digester used as soil fertilizer after aerobic com- 
posting. $\mathrm{Li}$ et al. (2018) detected an average microplastic concentration of $22.7 \pm 12.1 \times 10^{3}$ particles per kilogram of dry weight in 79 sewage sludge samples from 28 wastewater treatment plants in China. The total amount of microplastics that have already entered soil habitats is uncertain, but $\mathrm{Ng}$ et al. (2018) estimated that 2.3 to $63.0 \mathrm{Mg} \mathrm{ha}^{-1}$ microplastic loadings from biosolids have reached agroecosystems.

Properties of microplastics differ regarding their size, morphology, origin and chemical composition. A generally accepted definition for the term "microplastics" does not exist so far, although it is essential for industry, research and political decision makers. In several studies, microplastics are only defined as particles $<5 \mathrm{~mm}(5000 \mu \mathrm{m})$ and a contradistinction to nanoparticles is seldom given in environmental studies. Some environmental studies, however, classify microplastics into large ( 1 to $5 \mathrm{~mm})$ and small $(1 \mu \mathrm{m}$ to $1 \mathrm{~mm}$ ) particles (Wagner et al., 2014). The term "nanoplastic" and its definition is still controversial discussed. Gigault et al. (2018) classified nanoplastics and recommended $1 \mu \mathrm{m}$ as the upper size limit.

Microplastic particles are differentiated into primary microplastics (e.g., for abrasives, cosmetic additives or industrial resin pellets) and degraded secondary microplastics, which result from former larger plastic debris. Microplastic particles could be highly diverse regarding their morphology, leading to varying effects in environmental systems (Wagner et al., 2014).

More than 200 different types of plastics are known, which may have different properties, for example, regarding their reactivity or bioavailability in soil environments. Thus, differentiation of microplastics should not only be based on size but also regarding their chemical (e.g., hydrophobicity scales) and physical properties (e.g., morphology) that may affect physicochemical soil properties and soil biology. For instance, de Souza Machado et al. (2018) showed that $2 \%$ of the microplastic concentration in soil affects bulk density, water-holding capacity, hydraulic conductivity, soil aggregation, water stable aggregates and microbial activity. This comprehensive study elucidates the complexity of processes triggered by the presence of microplastic particles in the soil environment. Microglass is currently not part of the microplastics discussion, although glass is very resistant to corrosion or weathering and can be thought to be corrosion proof (Papadopoulos and Drosou, 2012). Microglass is used as a blasting abrasive, filling material and an additive in road markings. Thus, it enters the environment in similar ways to microplastics, for example, in sewage sludge or abrasive from roads. The effects on terrestrial ecosystems are as equally unknown as those of microplastics.

The difficulty of highly diverse study structures and test environments due to heterogenic material properties is already reported in related research disciplines like marine and freshwater ecology (Phuong et al., 2016; Rist and Hartmann, 2018). To create a standardized study structure in soil science, we highly recommend that future scientific studies dealing with the effect of artificial microparticles on soil flora and fauna use the definition and size. Furthermore, a detailed description of microparticle characteristics should be mandatory to show potential interactions between biotic or abiotic soil components and microparticles at different size scales.

The present study contributes to a deeper understanding of the impact of different types of microplastics and microglass $(\sim 100 \mu \mathrm{m})$ on the soil microbial community structure in an agricultural soil. To do this, different types of microplastics and microglass were added to arable soil and incubated for $80 \mathrm{~d}$. In order to identify possible shifts in the microbial community structure, we used phospholipid fatty analysis (PLFA). This study was guided by the following research questions:

1. Is it possible to observe distinct shifts in the microbial community due to the presence of microparticles?

2. Do different plastic material properties stimulate microbial groups in diverse ways?

3. Does microglass affect the microbial community in a similar way to microplastics?

\section{Material and methods}

\subsection{Soil sampling and incubation experiment}

Soil samples were taken on 11 March 2018 near Brachwitz $\left(51^{\circ} 31^{\prime} 46^{\prime \prime} \mathrm{N}, 11^{\circ} 52^{\prime} 41^{\prime \prime} \mathrm{E} ; 102 \mathrm{~m}\right.$ above sea level), $10 \mathrm{~km}$ northwest of Halle (Saale) (Saxony-Anhalt, Germany). The samples were randomly taken at four different spots (A, B, $\mathrm{C}$ and D) from the first $10 \mathrm{~cm}$ of an arable topsoil, in order to have four independent replicates, which served as basic substrate for the incubation experiment. The soil was immediately sieved $(<2 \mathrm{~mm})$ after sampling and divided into subsamples for further basic soil analytics. Subsample material used for incubation was stored at approximately $8^{\circ} \mathrm{C}$. The soil subsamples were set at a water content of $60 \%$ waterholding capacity (WHC) and preincubated for three weeks at $20^{\circ} \mathrm{C}$.

A respective amount of $1 \%(w / w)$ of polypropylene (PP), low-density polyethylene (LDPE), polystyrene (PS), polyamide 12 (PA12; Rompan, Remda-Teichel, Germany) and microglass (Kraemer Pigmente GmbH \& Co. KG, Aichstetten, Germany) was added to each independent soil replicate and stirred manually, for homogenization, with a glass stirring rod. This quantity is equal to $12.6 \mathrm{Mg}$ microparticles ha ${ }^{-1}$ (bulk density topsoil $-1.26 \mathrm{~g} \mathrm{~cm}^{-3}$ ) indicating the worst-case scenario. However, a study by Fuller and Gautam (2016) found similar contaminated soils close to industrial areas. In addition, control soil replicates were incubated without additives of microplastics or microglass. Due to the use of arable topsoil as the incubation substrate, a microplastic contamination cannot be excluded. However, due to the 
high microplastic loads used in this the experiment, a possible prior contamination is negligible. Microplastics were not pretreated to cause degradation (e.g., with ultraviolet radiation) in order to simulate primary microplastic particles in the soils. Incubation was performed in laboratory bottles for $80 \mathrm{~d}$ at $20^{\circ} \mathrm{C}$ without daylight. During this period, all bottles were opened weekly for $30 \mathrm{~s}$ in order to secure aerobic conditions. Furthermore, the total weight of each bottle was monitored. In the case of any weight loss, an equivalent amount of water was replenished to provide a constant water-holding capacity of $60 \%$. According to manufacturer specifications, the sizes of microplastic and microglass particles ranged between 90 and $100 \mu \mathrm{m}$. The microplastics used in this study are commonly used for daily products and cosmetics (bottle caps and drinking straws - PP; plastic bags, milk bottles and food packaging film - LDPE; disposable cups and packaging materials - PS; and inks and clothing - PA) and were detected in high amounts in sewage sludge of Lower Saxony (Mintenig et al., 2017; Shah et al., 2008).

\subsection{Soil basic properties}

For soil basic characterization, soil subsamples (not samples for incubation) were air dried and sieved ( $<2 \mathrm{~mm})$. Total carbon (TC) and total nitrogen (TN) analyses were carried out with a vario MAX cube CNS analyzer (Elementar Analysensysteme GmbH, Langenselbold, Germany). Electrical conductivity (EC) and $\mathrm{pH}$ values were analyzed by using suspensions of $0.01 \mathrm{M} \mathrm{CaCl}_{2}$ and distilled $\mathrm{H}_{2} \mathrm{O}$ at a soil solution ratio of 1 to 2.5 . Soil particle size distribution was measured in a suspension using a HELOS/KR laser diffractometer (Sympatec GmbH, Clausthal-Zellerfeld, Germany) equipped with a Quixel wet dispersion unit (Sympatec $\mathrm{GmbH}$, Clausthal-Zellerfeld, Germany). Before analysis, the sample material was treated with a dispersing agent $(0.2 \mathrm{M}$ tetra-Sodium diphosphate decahydrate). For the evaluation of the water-holding capacity, $10 \mathrm{~g}$ of soil was weighed into a plastic cylinder with a fine mesh at the bottom and placed into water. After $24 \mathrm{~h}$, the saturated samples were drained until the water release stopped, and they were weighed again for the calculation of the water-holding capacity. Soil subsamples used for the determination of soil basic properties were not used for the incubation experiment.

The soil chemical properties of the Chernozem topsoil (IUSS Working Group WRB, 2015) were as follows: total organic carbon (TOC) $28.6 \pm 1.8 \mathrm{~g} \mathrm{~kg}^{-1}$; total nitrogen (TN) $2.48 \pm 0.13 \mathrm{~g} \mathrm{~kg}^{-1} ; \mathrm{C}: \mathrm{N} 11.56 \pm 0.15$; EC $170 \pm$ $9 \mu \mathrm{S} \mathrm{cm}^{-1}$; and $\mathrm{pH}_{\mathrm{CaCl}_{2}} 5.13 \pm 0.02$. Proportions of clay, silt and sand were $7.0 \pm 0.2 \%, 58.5 \pm 3.6 \%$ and $34.5 \pm$ $3.7 \%$, respectively and the soil texture was classified as silt loam (FAO, 2006). The water-holding capacity was $0.218 \pm$ $0.005 \mathrm{~g}_{\mathrm{H}_{2} \mathrm{O}} \mathrm{g}_{\text {dry weight }}^{-1}$.

\subsection{Phospholipid fatty acid analysis}

Phospholipid fatty acid (PLFA) analyses were performed using a modified version of the Bligh and Dyer method (Frostegård et al., 1993). A total of $6 \mathrm{~g}$ of fresh soil were extracted with a single-phase trichloromethane-methanolcitrate buffer system $(1: 2: 0.8 ; v / v / v)$, and $19: 0$ was added as the first internal standard (IS1) to each sample for later quantification of the phospholipids. Extracts were centrifuged for $1 \mathrm{~min}$ at $4000 \mathrm{rpm}$. The supernatants were separated using a liquid-liquid extraction. Lipid fractionation was performed using a silica-based solid-phase extraction. The remaining phospholipid fractions of the samples and the external standards were treated with an alkaline saponification using $0.5 \mathrm{M}$ sodium hydroxide in methanol followed by a methylation with boron trifluoride in methanol (12\%). A liquid-liquid extraction, with saturated sodium chloride solution and hexane, was used to separate the organic phase, which contains the fatty acid methyl esters. For quality control, $5 \alpha$-cholestane was added as second internal standard (IS2) after the phase separation. Analytes were transferred with isooctane into gas chromatography (GC) autosampler vials and analyzed by a GC-2010 capillary gas chromatograph (Shimadzu Corporation, Tokyo, Japan) equipped with a Supelco SPB-5 fused silica capillary column $(30 \mathrm{~m} \times 0.25 \mathrm{~mm} \times 0.25 \mu \mathrm{m}$ film thickness $)$ and a flame ionization detector. All PLFA contents were corrected for dry mass due to the use of fresh soil for extraction. For this purpose, WHC was determined subsequent to the sample weighing.

Single PLFA were assigned to taxonomic groups according to following pattern: total fungi $-18: 2 \omega 6,9$ and $18: 1 \omega 9 \mathrm{c}$;

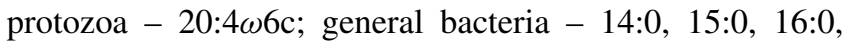
17:0 and 18:0; gram-positive bacteria - i14:0, a14:0, i15:0, a15:0, i16:0, a16:0, i17:0 and a17:0; gram-negative bacteria

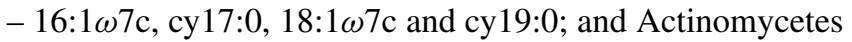
(ACT) - 10Me18:0 (Frostegård et al., 1993; Olsson et al., 1999; Zelles, 1999; Zelles et al., 1992). These biomarkers are not entirely specific for their taxonomic groups and therefore must be interpreted cautiously (Zelles, 1997). For total bacteria the sum of general, gram positive, gram negative and ACT was calculated. The sum of PLFA describes the sum of the measured contents of fungal-derived, bacterial-derived, protozoa and the unspecific PLFA markers of $16: 1 \omega 5 \mathrm{c}$ and 10Me16:0.

\subsection{Scanning electron microscopy (SEM)}

Microplastic samples were fixed on an object slide and coated with gold using a Q150R ES rotary-pumped sputter coater (Quorum Technologies Ltd., Laughton, United Kingdom) in a low-vacuum atmosphere. The scanning electron microscopy (SEM) images were taken with a Tabletop Microscope TM4000Plus (Hitachi Ltd., Tokyo, Japan). 


\subsection{Statistical analysis}

Statistical analysis and graphical design were carried out using $\mathrm{R}$ version 3.5.0 ( $\mathrm{R}$ Core Team, 2018). A prior test assumption of normally distributed data was examined using the Shapiro-Wilk test. Because of the mostly nonnormal distributed data, the Brown-Forsythe test was used for checking for homoscedasticity in the groups. The residuals of each linear model were checked graphically for homoscedasticity and normal distribution to validate the model performance. Because of the widespread heteroscedasticity and bad model performances, differences in PLFA marker contents between treatments of each taxonomic microbial group were statistically evaluated using the Kruskal-Wallis rank sum test. A Dunn's test was performed for multiple comparisons between the treatment levels in case of a significant $(p \leq 0.05)$ treatment effect in the Kruskal-Wallis test (Dunn, 1964). The Holm method was used to control the family-wise error rate caused by the pairwise multiple comparisons (Holm, 1979). Different lowercase letters were used to illustrate the significant differences between the homogeneous subsets, and the interquartile range of the boxplot whiskers is 1.5.

\section{Results}

\subsection{Morphology and size of microparticles}

The SEM images of the microplastics (PP, LDPE, PS and PA12) and microglass are shown in Fig. 1, illustrating the heterogenic morphology between, but also within, the same type of microplastic. Furthermore, according to the manufacturer, the specifications size of microplastics and microglass should range between 90 and $100 \mu \mathrm{m}$. Many particles are, however, much bigger (up to $200 \mu \mathrm{m}$ ) or smaller (down to $10 \mu \mathrm{m}$ ). In particular, LDPE, PA12 and PP have a slaglike structure that leads to pore formation, whereas PS has a plate-shaped structure with fringed or even sharp edges. Pointy and sharp edges are also shown for LDPE, PA12 and PP. In contrast, microglass particles appear, with a few exceptions, more regularly than the microplastic ones and could be described as microspheres.

\subsection{Impact of microplastics and microglass on soil microbial community structure}

The total PLFA contents do not show significant differences between single specific microparticles compared to the control (Fig. 2). Nevertheless, the PLFA contents of microglass, PP and LDPE in treated soil tend to increase compared to the control, with $11 \%, 19 \%$ and $28 \%$, respectively, whereas PA12 and PS show lower PLFA contents compared to the control by $32 \%$ and $11 \%$. The comparisons of single plastic types show that the PLFA contents of PA12 and PS are $89 \%$ and $43 \%$, respectively, which is significantly lower compared to LDPE (Fig. 2). A similar pattern is also observable in the treatment distribution of each group's PLFA content of bacteria and fungi, although the fungi show a more inexplicit pattern compared to bacteria. This might imply that positive and negative stimulations of the single microplastics affect bacteria and fungi in a similar way. Compared to the control, bacterial-derived PLFA contents show an increase in soil treated with microglass (19\%), PP (25\%) and LDPE (32\%). On the other hand, a decline in total bacteria has been determined in soil treated with PA12 $(-33 \%)$ and PS $(-11 \%$, Fig. 3). Fungal PLFA contents, however, show a smaller increase compared to the control with $9 \%$ (microglass), $15 \%$ (PP), $24 \%$ (LDPE) and a lower decrease of $-22 \%$ (PA12) and $-9 \%$ (PS; Fig. 3). The treatment effect variability of bacterial-derived PLFAs is multiple times higher compared to fungal-derived PLFAs. For instance, the highest positive median deviation of total bacterial-derived PLFAs to the control is $32 \%$ (LDPE), whereas the highest negative deviation is $33 \%$ (PA12). In contrast, the positive deviation of fungalderived PLFAs compared to the control is only $24 \%$ (LDPE) and negative deviation is only $22 \%$ (PA12; Fig. 3).

Regarding a whole comparison of all treatments, with the exception of protozoa, the increase in PLFA contents could be observed for all fungal and bacterial (negative, gram positive, ACT and general) groups when incubated with microglass, LDPE and PS (Fig. 3). The significantly lower PLFA contents of PA12 compared to LDPE are also shown continuously in all microbial groups (Fig. 3). In contrast to the fairly consistent pattern of the fungi and bacteria, protozoa show a different pattern. Protozoa PLFA contents decreased for all microplastics by up to $21 \%$ (LDPE) compared to the control (Fig. 3). PA12 and PP show a comparatively high data variability compared to the other treatments. Most interestingly, the PLFA content of protozoa was under the limit of determination for all replications incubated with microglass.

\section{Discussion}

High amounts of artificial soil impurities $(12.6 \mathrm{Mg} \mathrm{mi}-$ croplastics or glass ha ${ }^{-1}$ ) do not have a significant effect on soil microbial community structure within the incubation time of $80 \mathrm{~d}$. However, there is a conspicuous tendency that different types of microplastics may have promoting (LDPE; PP) or reducing the effects (PA12; PS) on soil microorganisms (Figs. 2 and 3). Furthermore, different plastics obviously have various effects on individual taxonomic groups, as indicated by the significantly lower values of treatment for PA12 and PS compared to LDPE (Figs. 2 and 3). As mentioned in Sect. 3.2, the variability of bacterial-derived PLFA is much higher than in fungal-derived PLFAs, which possibly indicates that bacteria are more susceptible to interference. However, this is not surprising because bacteria respond relatively quickly to environmental changes (e.g., changing water conditions, temperature, etc.), for example, due to their rapid reproduction rate (e.g., Fierer et al., 2003). 

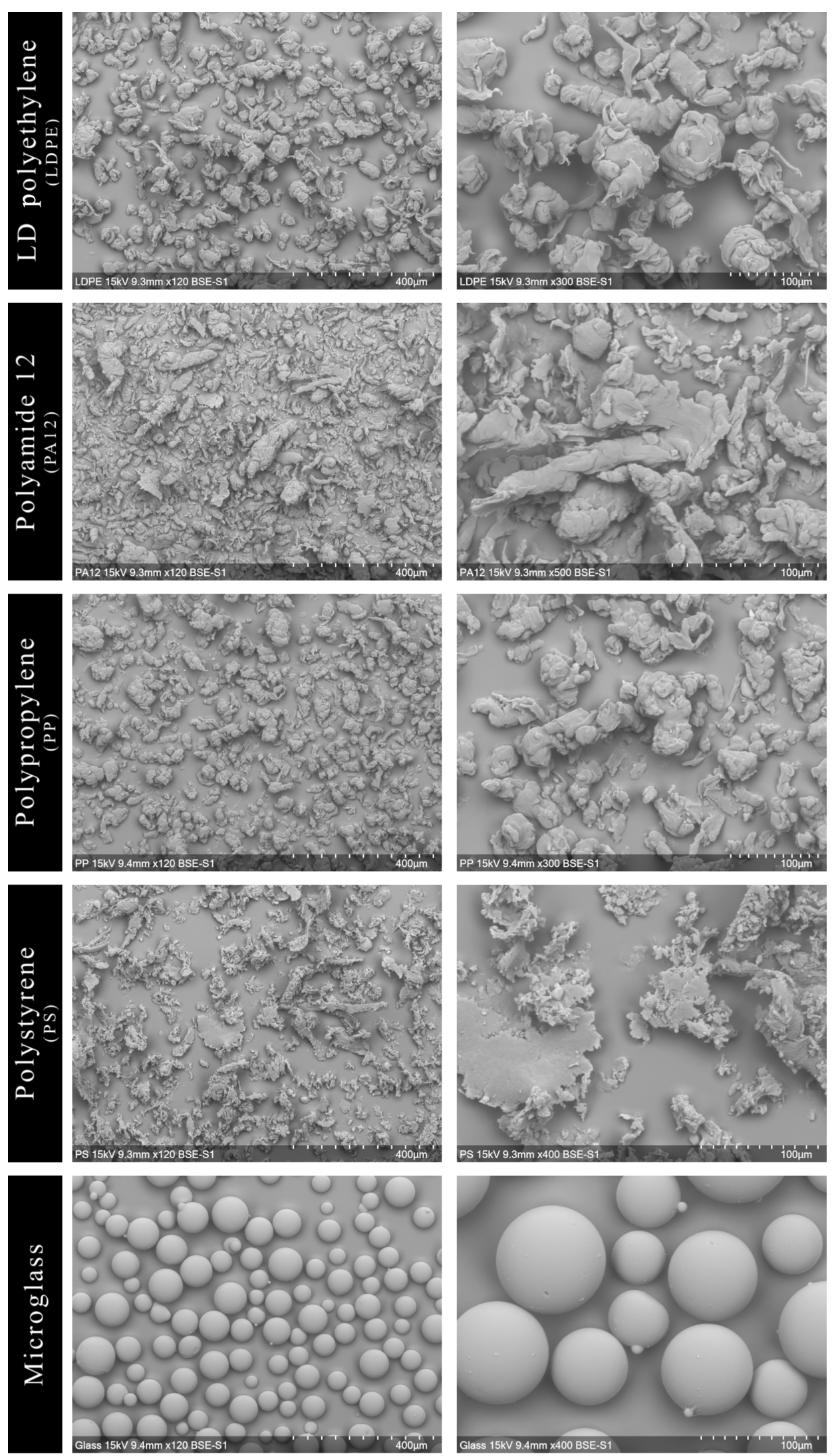

Figure 1. Heterogenic particle size distribution and morphology depending on the microparticle type visualized by the scanning electron microscopy (SEM). 


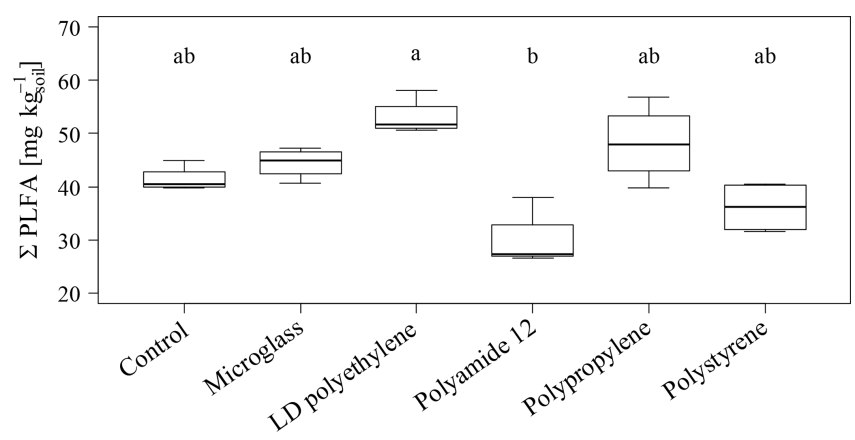

Figure 2. Sum of total phospholipid fatty acids as microbial markers in an incubated Chernozem after $80 \mathrm{~d}$. Different lowercase letters indicate significant differences between the treatments according to a multiple comparison with the Dunn's test $(n=4, p<0.05)$.

Reasons for missing the significant effects between microparticle treatments and the untreated control after $80 \mathrm{~d}$ may be found in the conscious choice of primary microplastics, which were not pretreated to cause a physical degradation (e.g., ultraviolet radiation). Subsequently, microplastics are mostly chemically inert during the experiment due to unaltered chemical and physical properties which, for example, prohibit the exposition of potential ecotoxic compounds. Nevertheless, the treatment of soil with different microparticles causes changes in microbial communities, albeit not significant. The observed effects are based on complex soilimpurity interactions, and studies dealing with the impact of microplastics on soil microbiology are still lacking (Rillig and Bonkowski, 2018; Zhang et al., 2019) and, to our best knowledge, published PLFA or even DNA-based studies are still missing.

However, de Souza Machado et al. (2018) investigated the microbial activity after the addition of different amounts of polyester and polyacrylic fibers and polyethylene fragments by measuring the enzyme activity with fluorescein diacetate (FDA). The study showed that polyester and polyacrylic fibers reduced microbial activity whereas the soil incubated with polyethylene fragments showed no clear tendency. The effects might be caused, for example, through changes in soil bulk density, water-holding capacity or aggregate changes (de Souza Machado et al., 2018). The reasons for the observed promoting and also inhibiting effects on microorganisms from different plastic types remain a matter of speculation, and further research is necessary to address these issues. The causes mentioned by de Souza Machado et al. (2018) are essential reasons that affect soil microbiology.

Nevertheless, the morphology and surface properties of microplastics should not be underestimated. The slag-like structure of LDPE, PA12 and PP form wrinkles and pores (Fig. 1) and may act as a habitat for soil microorganisms. This, in turn, may have a promoting effect on the microbial community composition of soil as seen with pore-rich soil additives, for example, such as charcoal (biochar). For instance, fungal hyphae or bacteria penetrate the pores and wrinkles and are protected from predators (Lehmann et al., 2011; Thies and Rillig, 2009). Furthermore, McCormick et al. (2014) showed that microplastic particles could act as a habitat for bacteria in rivers. Umamaheswari et al. (2014) found fungi hyphae from Penicillium sp., Fusarium sp. and Aspergillus sp., which colonized and grew on the surface of soil-buried PS after $70 \mathrm{~d}$. The potential colonization of microorganisms on the surface of LDPE was clearly reviewed by Kumar Sen and Raut (2015), who also mentioned the penetration of the microplastic surface by fungi hyphae. Similar colonization of bacteria was reported by Harrison et al. (2014), who found the rapid attachment of microorganisms onto LDPE microplastics within coastal marine sediments after $14 \mathrm{~d}$. In sum, LDPE seems to benefit the bacterial and fungal colonization. Both bacteria and fungi tended to increase their populations in our experiment. LDPE may also act as habitat and a carbon source. The extent of these functions is mostly controlled by abiotic factors, for example, ultraviolet irradiation and temperature (Kumar Sen and Raut, 2015). Thus, the provided habitat seems to be the most important factor for enhanced PLFA in our experiment because abiotic factors were either excluded (no ultraviolet irradiation) or kept constant (stable temperature at $20^{\circ} \mathrm{C}$ ). However, colonization on microplastic surfaces after incubation was not determined in this experiment, and currently it is still uncertain if colonized microplastic surface areas could also act as a hotbed for extensive soil colonization. Furthermore, it remains uncertain why PA12 seems to inhibit microorganisms in this experiment despite having similar surface properties to, for example, LDPE, which tends to promote the microorganisms. According to Galloway et al. (2017), organic compounds, nutrients and pollutants can accumulate on microplastic surface in aquatic ecosystems. It can be assumed that this also occurs in terrestrial ecosystems such as soil environments. Furthermore, it is conceivable that humic substances also accumulate on microplastic surfaces, leading to an increased colonization of specific microorganisms and, consequently, to the formation of a bacterial biofilm. The accumulation of nutrients and water on a surface is the precondition for the formation of biofilms consisting of extracellular polymeric substances derived from bacteria (Flemming and Wingender, 2010). The formation of biofilms may occur within three weeks, as shown by Lobelle and Cunliffe (2011) who investigated the surface of PE particles in a marine environment. Due to the constant (water) conditions in this study, the formation of biofilms on microplastic surfaces cannot be excluded at least on LDPE and PP particles and microglass, indicating the promoting effects on soil microorganisms reflected by increased PLFA contents. Future research on the role of artificial microparticles in soil microcosms is urgently needed to clarify potential risks and intensities of soil microbiological disturbance by microplastics due to promoting the colonization of specialized (and harmful) microorganisms, toxicity due to released harmful chemicals or direct damage 

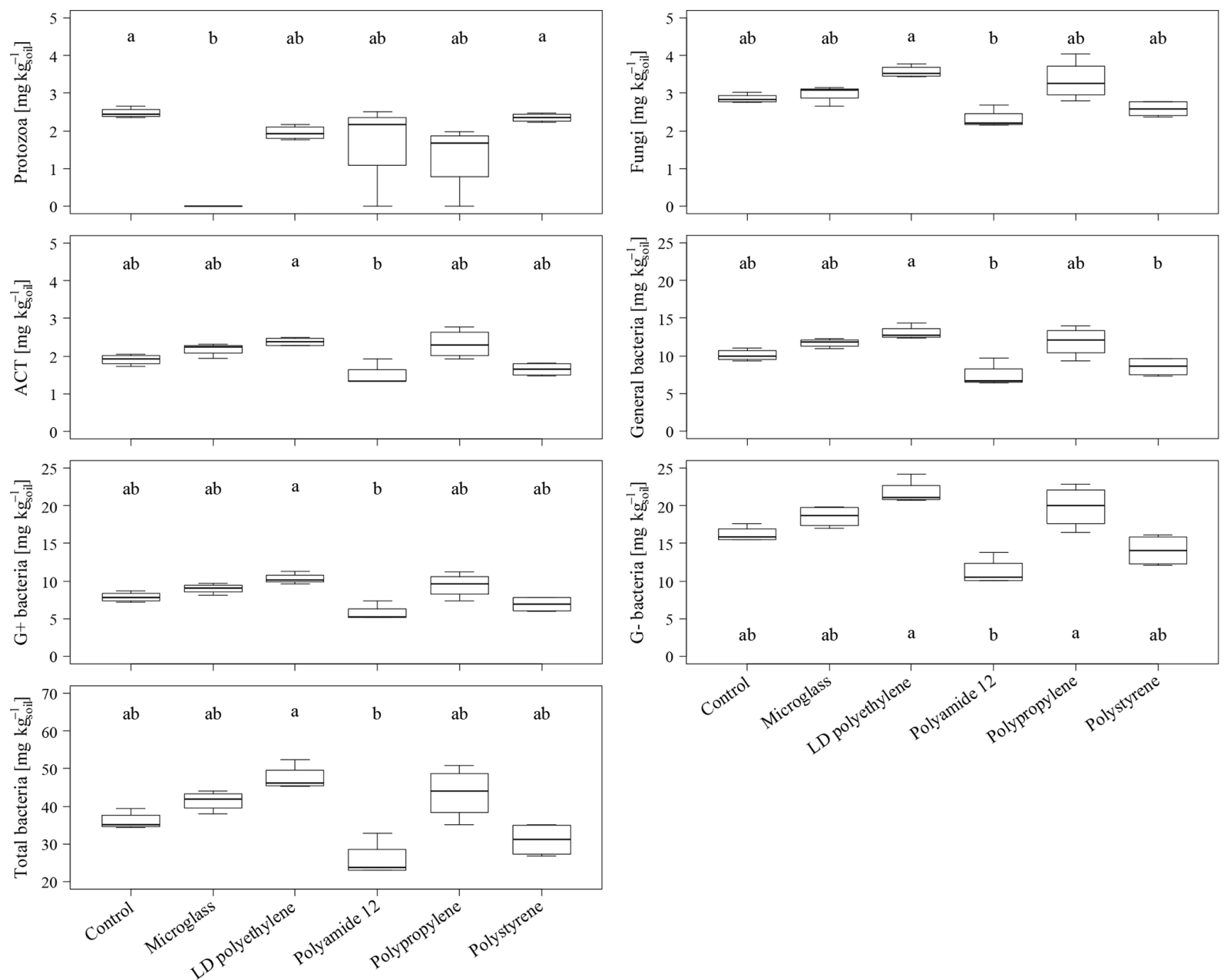

Figure 3. Microbial-derived phospholipid fatty acid contents of the individual taxonomic groups of an incubated Chernozem after $80 \mathrm{~d}$. Different lowercase letters indicate significant differences in the treatments according to a multiple comparison with the Dunn's test $(n=4$, $p<0.05)$. Please note the varying ordinate scales.

after entering the microorganism as secondary nanoparticles (Lu et al., 2019).

Besides the morphology of the microplastic, its surface chemistry has effects on soil physicochemical processes. In comparison to LDPE, PP and PS, which show hydrophobic characteristics, PA12 combines hydrophobic and hydrophilic surface groups (Schmidt et al., 2015), whereas microglass has a hydrophilic surface. A study by Marangoni et al. (2018) showed that glass microspheres $(4,7-10$ and $30-50 \mu \mathrm{m}$; microglass addition of $1 \% \mathrm{v} / \mathrm{v}-5 \% \mathrm{v} / \mathrm{v}$ ) reduced the mobility of water reflected in a large decrease of the spin-spin relaxation time of water protons, decreased the self-diffusion coefficient of water molecules, lowered water activity and strengthened $\mathrm{OH}$ bonds. The study further showed that glass microspheres have an inhibiting effect on Escherichia coli growth and the germination of Medicago sativa seeds. In our experiment, an inhibiting effect of microglass could not be shown for most microorganisms, with the exception of proto- zoa (Fig. 3). Based on the results by Marangoni et al. (2018), it is conceivable that protozoa respond in a similar way to the presence of microglass, such as Escherichia coli. Nevertheless, these harmful effects of microglass particles on protozoa observed in our study are surprising because this indicates that, for example, sand grains in soil, which consist of $\mathrm{SiO}_{2}$, may also have inhibitory effects on protozoa. To our best knowledge, no studies were performed in order to investigate this question.

Another important fact relates to the heterogeneity of microplastics. The wide variance between the several types of plastics and the heterogeneity of different sources prevent a generalization of scientific results. For example, Cao et al. (2017) visualized polystyrene using SEM. This image of PS differs strongly from the plastic used in our study. The way of producing the pathway to the environment and the degradation status of microplastics play an important role in evaluating the behavior of microplastics in soil or 
other environments. Furthermore, it remains ambiguous as to whether primary microplastics added to soils cause similar effects compared to secondary microplastics, which result from the decomposition of larger plastic debris. Depending on the parent plastic material and environmental variables, highly diverse plastic surfaces result from uncontrolled surface modification due to decomposition processes. This fact is already known from the comparison of primary and secondary nanoplastics properties (Gigault et al., 2018), and it is especially noteworthy that already emitted macro- and microplastics will degrade in terrestrial ecosystems right up to the nanoscales.

Nevertheless, it should be borne in mind that PLFA analyses and laboratory experiments always generate limited results. A fast change in the PLFA pattern only allows for a determination of the actual state of the microbial community structure and it is unreliable to use a single PLFA biomarker for taxa detection, which is feasible with deoxyribonucleic acid (DNA) analyses. But compared to gene sequencing or other DNA analyses, PFLA biomarker analysis is faster and cheaper (Frostegård et al., 2011). Another problem may be the transferability of the results generated at the laboratory scale under ideal conditions (well-known homogenous plastic fabrics used as treatments, simplified and controllable regimes, no rhizosphere, etc.). Also, the single addition of high amounts of microplastics does not reflect the ordinary way in which microplastics enter an ecosystem. The accumulation of plastic particles in soils is a long and gradual process rather than a single event and does not trigger sudden environmental impacts (Rillig et al., 2019). Thus, this first study should only serve as a basic work which stimulates future microbial studies that deal with microparticles in soils or sediments. So, further research is needed to link the laboratory and environmental conditions and to enhance the environmental relevance of microplastic research. High amounts were chosen to show worst-case scenario effects on highly contaminated places (industrial areas or floodplains in vicinity of urban areas). On the other hand, agricultural land is treated regularly with compost, sewage sludge and other microplastics-containing soil amendments or plastic mulches are used in vegetable production. Due to their recalcitrance, plastics tend to accumulate in the soil. So, a worst-case scenario is able to illustrate future soil statuses at an undefined timescale.

\section{Conclusions}

This study aimed to address the question of whether high amounts of microplastics and microglass have effects on the soil's microbial community structure by using PLFAs as microbial markers. High amounts were added to soil in order to show a worst-case scenario in highly contaminated soils (e.g., industrial areas or floodplains in vicinity of urban areas). On the other hand, agricultural land is treated regu- larly with compost, sewage sludge and other microplasticscontaining soil amendments. Furthermore, plastic mulches used for fruit and vegetable production are further sources of microplastics in soils. Due to its high recalcitrance, plastic tends to accumulate in the soil. Thus, our worst-case scenario may illustrate future soil statuses at an undefined timescale. The use of microbial markers in laboratory incubation experiments describing microbial soil communities always acts as a simplification of complex natural environmental systems. This study provides the first insights into soil microcosms being disturbed by different microparticles. The results provide hints that, after $80 \mathrm{~d}$ of incubation, microorganisms are either promoted or inhibited depending on the type of the impurities. Different microplastic types seem to have contrary effects on soil microorganisms, depending on the origin and the properties of the plastics, which influence the morphological and chemical appearance of the microplastics. On the other hand, microglass even seems to be highly toxic for protozoa. Within this study we cannot clarify why bacteria and protozoa show different reactions to quartz glass microparticles. Changes in soil microbiology induced by plastic pollution have unexpected consequences for soil ecosystems. This study should therefore be considered as basis for further research which is urgently needed in order to understand the long-term consequences of microplastics in soils and other terrestrial ecosystems.

Data availability. All data compiled in this study are published in the figures. Detailed primary data and underlying research are available on request from the corresponding author.

Author contributions. KW conceptualized and carried out the experiment. Laboratory work was performed by KW and SP. Statistical analysis and data visualization was carried out by SP. KW prepared the paper with contributions from SP.

Competing interests. The authors declare that they have no conflict of interest.

Acknowledgements. We acknowledge Bruno Glaser (Department of Soil Biogeochemistry, Martin Luther University, HalleWittenberg) for providing the Soil Biogeochemistry laboratories for the PLFA analyses. We are grateful to Aline Brosch for supporting the incubation experiment. Furthermore, we thank Tobias Bromm (Department of Soil Biogeochemistry, Martin Luther University, Halle-Wittenberg) for supporting the GC measurements. We also thank Gregor Borg (Department of Economic Geology and Petrology, Martin Luther University, Halle-Wittenberg) and his staff, Andreas Kamradt and Tim Rödel, for providing the SEM. Finally, we are grateful to the anonymous reviewers and editor for their critical and detailed comments. 
Review statement. This paper was edited by Jeanette Whitaker and reviewed by two anonymous referees.

\section{References}

Bläsing, M. and Amelung, W.: Plastics in soil: Analytical methods and possible sources, Sci. Total Environ., 612, 422-435, https://doi.org/10.1016/j.scitotenv.2017.08.086, 2018.

Cao, D., Wang, X., Luo, X., Liu, G., and Zheng, H.: Effects of polystyrene microplastics on the fitness of earthworms in an agricultural soil, IOP Conf. Ser. Earth Environ. Sci., 61, 012148, https://doi.org/10.1088/1755-1315/61/1/012148, 2017.

de Souza Machado, A. A., Lau, C. W., Till, J., Kloas, W., Lehmann, A., Becker, R., and Rillig, M. C.: Impacts of Microplastics on the Soil Biophysical Environment, Environ. Sci. Technol., 52, 96569665, https://doi.org/10.1021/acs.est.8b02212, 2018.

Dunn, O. J.: Multiple Comparisons Using Rank Sums, Technometrics, 6, 241-252, https://doi.org/10.1080/00401706.1964.10490181, 1964.

FAO: Guidelines for Soil Description, Fourth Edition, Rome, Italy, available at: http://www.fao.org/3/a0541e/A0541E.pdf (last access: 4 February 2019), 2006.

Fierer, N., Schimel, J. P., and Holden, P. A.: Influence of DryingRewetting Frequency on Soil Bacterial Community Structure, Microbiol. Ecol., 45, 63-71, https://doi.org/10.1007/s00248002-1007-2, 2003.

Flemming, H.-C. and Wingender, J.: The biofilm matrix, Nat. Rev. Microbiol., 8, 623-633, https://doi.org/10.1038/nrmicro2415, 2010.

Frostegård, Å., Bååth, E., and Tunlio, A.: Shifts in the structure of soil microbial communities in limed forests as revealed by phospholipid fatty acid analysis, Soil Biol. Biochem., 25, 723-730, https://doi.org/10.1016/0038-0717(93)90113-P, 1993.

Frostegård, Å., Tunlid, A., and Bååth, E.: Use and misuse of PLFA measurements in soils, Soil Biol. Biochem., 43, 1621-1625, https://doi.org/10.1016/j.soilbio.2010.11.021, 2011.

Fuller, S. and Gautam, A.: A Procedure for Measuring Microplastics using Pressurized Fluid Extraction, Environ. Sci. Technol., 50, 5774-5780, https://doi.org/10.1021/acs.est.6b00816, 2016.

Galloway, T. S., Cole, M., and Lewis, C.: Interactions of microplastic debris throughout the marine ecosystem, Nat. Ecol. Evol., 1, 0116, https://doi.org/10.1038/s41559-017-0116, 2017.

Gigault, J., ter Halle, A., Baudrimont, M., Pascal, P.-Y., Gauffre, F., Phi, T.-L., El Hadri, H., Grassl, B., and Reynaud, S.: Current opinion: What is a nanoplastic?, Environ. Pollut., 235, 10301034, https://doi.org/10.1016/j.envpol.2018.01.024, 2018.

Harrison, J. P., Schratzberger, M., Sapp, M., and Osborn, A. M.: Rapid bacterial colonization of low-density polyethylene microplastics in coastal sediment microcosms, BMC Microbiol., 14, 1-15, https://doi.org/10.1186/s12866-014-0232-4, 2014.

Holm, S.: A Simple Sequentially Rejective Multiple Test Procedure, Scand. J. Stat., 6, 65-70, 1979.

Hurley, R. R. and Nizzetto, L.: Fate and occurrence of micro(nano)plastics in soils: Knowledge gaps and possible risks, Curr. Opin. Environ. Sci. Heal., 1, 6-11, https://doi.org/10.1016/j.coesh.2017.10.006, 2018.

Hurley, R. R., Lusher, A. L., Olsen, M., and Nizzetto, L.: Validation of a Method for Extracting Microplastics from Complex,
Organic-Rich, Environmental Matrices, Environ. Sci. Technol., 52, 7409-7417, https://doi.org/10.1021/acs.est.8b01517, 2018.

IUSS Working Group WRB: World Reference Base for Soil Resources 2014, International soil classification system for naming soils and creating legends for soil maps, Rome, Italy, available at: http://www.fao.org/3/i3794en/I3794en.pdf (last access: 10 May 2018), 2015.

Kumar Sen, S. and Raut, S.: Microbial degradation of low density polyethylene (LDPE): A review, J. Environ. Chem. Eng., 3, 462473, https://doi.org/10.1016/j.jece.2015.01.003, 2015.

Kyrikou, I. and Briassoulis, D.: Biodegradation of Agricultural Plastic Films: A Critical Review, J. Polym. Environ., 15, 125150, https://doi.org/10.1007/s10924-007-0053-8, 2007.

Lehmann, J., Rillig, M. C., Thies, J., Masiello, C. A., Hockaday, W. C., and Crowley, D.: Biochar effects on soil biota - A review, Soil Biol. Biochem., 43, 1812-1836, https://doi.org/10.1016/j.soilbio.2011.04.022, 2011.

Li, X., Chen, L., Mei, Q., Dong, B., Dai, X., Ding, G., and Zeng, E. Y.: Microplastics in sewage sludge from the wastewater treatment plants in China, Water Res., 142, 75-85, https://doi.org/10.1016/j.watres.2018.05.034, 2018.

Lobelle, D. and Cunliffe, M.: Early microbial biofilm formation on marine plastic debris, Mar. Pollut. Bull., 62, 197-200, https://doi.org/10.1016/j.marpolbul.2010.10.013, 2011.

Lu, L., Luo, T., Zhao, Y., Cai, C., Fu, Z., and Jin, Y.: Interaction between microplastics and microorganism as well as gut microbiota: A consideration on environmental animal and human health, Sci. Total Environ., 667, 94-100, https://doi.org/10.1016/j.scitotenv.2019.02.380, 2019.

Marangoni, A. G., Al-Abdul-Wahid, M. S., Nicholson, R., Roma, A., Gravelle, A. J., De Souza, J., Barbut, S., and Spagnuolo, P. A.: Water immobilization by glass microspheres affects biological activity, Sci. Rep., 8, 9744, https://doi.org/10.1038/s41598-01828123-4, 2018.

McCormick, A., Hoellein, T. J., Mason, S. A., Schluep, J., and Kelly, J. J.: Microplastic is an Abundant and Distinct Microbial Habitat in an Urban River, Environ. Sci. Technol., 48, 1186311871, https://doi.org/10.1021/es503610r, 2014.

Mintenig, S. M., Int-Veen, I., Löder, M. G. J., Primpke, S., and Gerdts, G.: Identification of microplastic in effluents of waste water treatment plants using focal plane array-based microFourier-transform infrared imaging, Water Res., 108, 365-372, https://doi.org/10.1016/j.watres.2016.11.015, 2017.

Ng, E.-L., Huerta Lwanga, E., Eldridge, S. M., Johnston, P., Hu, H.-W., Geissen, V., and Chen, D.: An overview of microplastic and nanoplastic pollution in agroecosystems, Sci. Total Environ., 627, 1377-1388, https://doi.org/10.1016/j.scitotenv.2018.01.341, 2018.

Olsson, P. A., Thingstrup, I., Jakobsen, I., and Bååth, E.: Estimation of the biomass of arbuscular mycorrhizal fungi in a linseed field, Soil Biol. Biochem., 31, 1879-1887, https://doi.org/10.1016/S0038-0717(99)00119-4, 1999.

Papadopoulos, N. and Drosou, C.-A.: Influence of weather conditions on glass properties, J. Univ. Chem. Technol. Metall., 47, 429-438, 2012.

Phuong, N. N., Zalouk-Vergnoux, A., Poirier, L., Kamari, A., Châtel, A., Mouneyrac, C., and Lagarde, F.: Is there any consistency between the microplastics found in the field and those 
used in laboratory experiments?, Environ. Pollut., 211, 111-123, https://doi.org/10.1016/j.envpol.2015.12.035, 2016.

R Core Team: R: A Language and Environment for Statistical Computing, available at: http://www.r-project.org, last access: 11 December 2018.

Rillig, M. C. and Bonkowski, M.: Microplastic and soil protists: A call for research, Environ. Pollut., 241, 1128-1131, https://doi.org/10.1016/j.envpol.2018.04.147, 2018.

Rillig, M. C., de Souza Machado, A. A., Lehmann, A., and Klümper, U.: Evolutionary implications of microplastics for soil biota, Environ. Chem., 16, 3-7, https://doi.org/10.1071/EN18118, 2019.

Rist, S. and Hartmann, N. B.: Aquatic Ecotoxicity of Microplastics and Nanoplastics: Lessons Learned from Engineered Nanomaterials, in: Freshwater Microplastics?: Emerging Environmental Contaminants?, edited by: Wagner, M. and Lambert, S., Springer International Publishing, Chamber, 25-49, 2018.

Schmidt, J., Sachs, M., Fanselow, S., Wirth, K.-E., Peukert, W., and Kolberg, S.: Funktionalisierung von Polymermaterialien für Laserstrahlschmelzverfahren, in: Neue Entwicklungen in der Additiven Fertigung, Springer Vieweg, Berlin, Heidelberg, 25-40, 2015.

Shah, A. A., Hasan, F., Hameed, A., and Ahmed, S.: Biological degradation of plastics: A comprehensive review, Biotechnol. Adv., 26, 246-265, https://doi.org/10.1016/j.biotechadv.2007.12.005, 2008.

Thies, J. E. and Rillig, M. C.: Characteristics of Biochar: Biological Properties, in: Biochar for Environmental Management: Science and Technology, edited by: Lehmann, J. and Joseph, S., Earthscan Ltd., London, 85-105, 2009.

Umamaheswari, S., Murali, M., and Thiyagarajan, R.: Role of Fungi Inhabiting Soil, Cow Dung and Sewage in Degrading Polyethylene Terephthalate and Polystyrene Foam, J. Pure Appl. Microbiol., 8, 2465-2471, 2014.
Wagner, M., Scherer, C., Alvarez-Muñoz, D., Brennholt, N., Bourrain, X., Buchinger, S., Fries, E., Grosbois, C., Klasmeier, J., Marti, T., Rodriguez-Mozaz, S., Urbatzka, R., Vethaak, A. D., Winther-Nielsen, M., and Reifferscheid, G.: Microplastics in freshwater ecosystems: what we know and what we need to know, Environ. Sci. Eur., 26, 1-9, https://doi.org/10.1186/s12302-014-0012-7, 2014.

Weithmann, N., Möller, J. N., Löder, M. G. J., Piehl, S., Laforsch, C., and Freitag, R.: Organic fertilizer as a vehicle for the entry of microplastic into the environment, Sci. Adv., 4, 1-7, https://doi.org/10.1126/sciadv.aap8060, 2018.

Zelles, L.: Phospholipid fatty acid profiles in selected members of soil microbial communities, Chemosphere, 35, 275-294, https://doi.org/10.1016/S0045-6535(97)00155-0, 1997.

Zelles, L.: Fatty acid patterns of phospholipids and lipopolysaccharides in the characterisation of microbial communities in soil: a review, Biol. Fertil. Soil., 29, 111-129, https://doi.org/10.1007/s003740050533, 1999.

Zelles, L., Bai, Q. Y., Beck, T., and Beese, F.: Signature fatty acids in phospholipids and lipopolysaccharides as indicators of microbial biomass and community structure in agricultural soils, Soil Biol. Biochem., 24, 317-323, https://doi.org/10.1016/00380717(92)90191-Y, 1992.

Zhang, S., Wang, J., Liu, X., Qu, F., Wang, X., Wang, X., Li, Y., and Sun, Y.: Microplastics in the environment: A review of analytical methods, distribution, and biological effects, TrAC Trends Anal. Chem., 111, 62-72, https://doi.org/10.1016/j.trac.2018.12.002, 2019. 